\title{
Reducing the Effects of Multiple Access Interference Signals Using Two Parallel Linear Antenna Array Based MVDR Beam former Technique
}

\author{
Suhail Najm Shahab \\ Department of Electronics Technique, AlHawija Technical Institute, \\ Northern Technical University, Kirkuk, Iraq \\ drsuhel@ntu.edu.iq
}

\begin{abstract}
The wireless communication system is increasingly becoming a vital factor worldwide nowadays. The performance of the wireless telecommunication system is sensitive to interference signals, which can be caused system degradation. The Beam forming technology has received much attention in the past decade due to interference and jamming signals reduction in the wireless communication system. Minimum Variance Distortion less Response (MVDR) is attracting widespread interest due to its ability to suppress interfering signals. In this paper, the MVDR beam former is applied on a parallel uniform linear array arraignment to reduce the effects of interference signals. The present paper aims to examine the behavior of the MVDR beam former in a 2D manner to illustrate the effects of element numbering on output beam pattern and SINR. Simulation outcomes show a notable correlation between the MVDR technique and the parallel uniform linear array geometry. The numerical result confirms that an increasing number of array elements leads to improve the overall system performance in terms of output SINR. The most remarkable correlation is a $1 \mathrm{~dB}$ increase in the SINR as adding one element to the array geometry. The findings of this work demonstrate the advantages of using the beam forming technique to reducing the effects of interference sources. Future work will look into side lobe level minimization.
\end{abstract}

Keywords: Antenna Array, Beam former, MVDR, SINR, Smart Antenna.

\section{Introduction}

Nowadays, the number of smart devices worldwide used by human beings or machine-to-machine communication systems is increased dramatically. By the year 2023 and according to Cisco, over 70\% of the world's population will have access to moveable devices in addition to that, half of the machine-to-machine smart device connections will be also connected $[1,2]$. Because of this huge amount of connected devices, a smart antenna system will be required. The smart antenna system is one of the talented technologies that can increase the ability of wireless communication systems by forcefully reducing the multipath component and co-channel interference and hence the number of users in a whole network can be increased [3]. Two major issues are concerned with the smart antenna direction of arrival (DOA) estimation and beam forming $[4,5]$. Smart antenna systems based on different antenna array arrangements are implemented in many studies in $1 \mathrm{D}$ and 2D dimensions. For instance, a study has been performed by [5] on 2D DOA estimation by using a parallel uniform linear array-based ESPRIT technique. The suggested method yields a perfect azimuth and elevation angles estimation in 2D DOA estimation effectively. More recent attempts have been made by [6] aimed to improve the 2D DOA estimation accuracy based on 3 parallel large space uniform linear arrays to resolve the ambiguity problem. The authors in [6] found that the proposed method has low complexity to the covariance matrix calculation. In [7], the researchers use a parallel nested array to find the 2D directional of arrival estimation based on the sparse Bayesian method. The proposed procedure 
reduces the size of the data noise and sparse dictionary. The outcomes found that the number of DOA signals estimated by the proposed system is much larger than the number of sensors. On the other hand, the beam forming technique gives an ability to decrease errors by transmitting signals to the desired angle and placing null to the interference sources. Minimum Variance Distortion less Response (MVDR) is a beam former technique that is capable to reduce the power of interference and noise signals without distorting the desired signal. In our previous work, many efforts have been conducted to assess the MVDR performance using linear, rectangular, and circular antenna arrays in $1 \mathrm{D}$ and 2D configurations [8-11]. The assessment found uniform linear array as 1D geometry based MVDR beam former gives accurate main beam steered to the desired user angle with null placed to the undesired signals than uniform rectangular array and uniform circular array. MVDR beam formers have been used for numerous purposes and applications such as radar, sonar, and communications to name a few. For example, a study proposed by has been used the MVDR beam former to diagnose images of breast cancer [12]. The study based a real-time test-bed to detect a microwave image using a wideband array of antennas and a breast phantom composite model. The conducted results found the proposed method-based MVDR algorithm shows its validity to diagnoses images of breast cancer. Also, another instance was suggested for speech enhancement by [13]. The suggested work used the MVDR technique to separate the desired speech from other interfering sources. A deep learning method was proposed that combined the MVDR with a neural network framework to reduce the effects of the residual noise while keeping the real speech signal distortion less. It was found a good speech separation system from this combine significantly reduces the residual noise against several new speech separation systems. Interference mitigation is one of the challenges of wireless communication systems. Antenna arrays with beam forming systems were used to eliminate the effects of interference signals. More recent work has been presented to suppress the narrowband and wideband interferences by [14]. As the number of array elements increases with a limited number of data samples is a big challenge due to high computational complexity. Here in [14], an artificial intelligence algorithm is employed to find the weight vector solution with superior solution-based linear antenna array geometry. Throughout this study, the simulation outcome obtained maximum SINR by the weight vector estimated from the neural process. In short, the interference source has big effects on the wireless system by reducing the number of users and the quality of signal served to the wanted user. In this paper, the performance of the MVDR beam former is evaluated based on two parallel uniform linear antenna arrays in a 2D manner to reduce the effects of the interference signals on wireless telecommunication. Beam pattern accuracy and output signal-to-interference-plus-noise ratio (SINR) is the two evaluation criteria that have been selected to evaluate the MVDR performance. The follows of the paper is organized as: The details of the MVDR beam former have been pointed out in Section 2 . Section 3 shows the simulation results of the proposed work. The last section highlighted the conclusion of MVDR based on two parallel linear arrays. 


\section{System Model \& MVDR Beam former}

One of the most sought aspects of wireless communication is the ability to provide consumers with dependable connectivity. Because the majority of users are not stationary and frequently change their location, the system must be able to track without losing the connection of the real user signal. Here, the system model for a parallel antenna array based MVDR beam former is described. First, the design of the linear antenna array model for the overall signal on the antenna elements and problem geometry will be given. Consider an antenna array consist of two-parallel uniform linear arrays with M/N omnidirectional sensors on the $\mathrm{x}$ - and $\mathrm{y}$-axis, respectively. The transmission source is coming from the far-field with a plane waves signal model is considered. Figure 1 is described a general research framework of this study that proposed for reducing the interference effects on the wireless communication system.

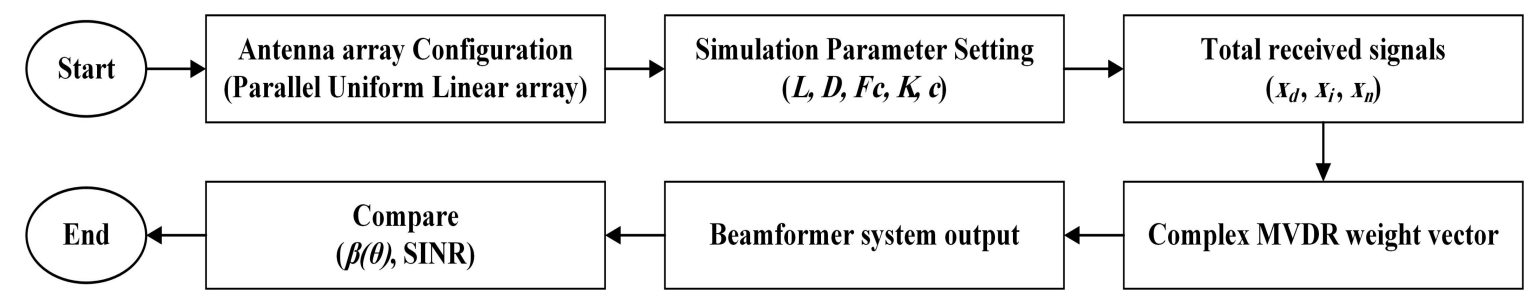

Figure 1: General research framework of the MVDR beam former

In an array of M-by-N elements, each element records a signal that contains the desired signal $\left(x_{d}\right)$, the interference signal $\left(x_{i}\right)$, and the noise from each sensor $\left(x_{n}\right)$. The total received signal $(X)$ at $k t h$ snapshot can be written as:

$$
X(K)=x_{d}(k) \cdot a_{d}\left(\theta_{d}\right)+x_{i}(k) \cdot a_{i}\left(\theta_{i}\right)+x_{n}
$$

Where $a_{d}\left(\theta_{d}\right), a_{i}\left(\theta_{i}\right)$ denote the array steering vector for the desired signal and the interference signals respectively. The $a(\theta)$ is a complex vector that can be given as [15]:

$$
a(\theta)=\left[\begin{array}{lllll}
1 & e^{-j q D \sin (\theta)} & e^{-j q 2 D \sin (\theta)} & \ldots & e^{-j q(L-1) D \sin (\theta)}
\end{array}\right]
$$

Where $q, \lambda, D, \theta$ denotes free-space wavenumber equal to $2 \pi / \lambda$, the free-space wavelength, the distance between neighboring antenna elements, azimuth angle with a scan range of $-\pi / 2$ to $\pi / 2$, respectively. Here, the undesired signals consist of an interference signal plus the additive White Gaussian noise. On the other side, the array output signal at $k t h$ data snapshot is composed as:

$$
Y(K)=X(K) \cdot w_{M V D R}^{H}
$$

Where $w$ is the complex weight vector for the MVDR beam former and $(.)^{\mathrm{H}}$ denotes Hermitian transpose (conjugate transpose). The MVDR beam former tries to maintain unity gain in the direction of the look angle while place nulls in the undesired signal angles. The MVDR calculate the weight vector by solving the following minimization equations through a unity gain restraint: 


$$
\begin{gathered}
w_{M V D R}=\arg \min _{w} E\left\{|Y(K)|^{2}\right\} \\
\min _{w} w^{H} \cdot R \cdot w \quad \text { subject to } w^{H} \cdot a_{d}\left(\theta_{d}\right)=1
\end{gathered}
$$

Where $E($.$) denotes the expectation operator of the beam former output and R$ is denote the array covariance matrix. The sampled covariance matrix for $K$ data snapshots is defined as:

$$
\hat{R}=1 / L \sum_{l=0}^{L-1}\left(X_{l}, X_{l}^{H}\right)
$$

Where $L$ denotes the number of array elements. The MVDR weights constrain in equations (5) are solved using Lagrange multipliers [5], yielding the following result:

$$
w=\hat{R}^{-1} a\left[a^{H} \hat{R}^{-1} a\right]^{-1}
$$

To assessing the MVDR performance in the beam former system. The beam pattern $\beta(\theta)$ is created by forming a beam in the accessible search region of $0^{\circ}$ to $180^{\circ}$ and displaying the related energy levels as assessment criteria. The array's beam pattern may be described as follows for the beam former system:

$$
\beta(\theta)=\sum_{l=0}^{L-1} w_{l}^{H} e^{-j \tau_{\theta, l}}
$$

Lastly, the SINR is defined as the ratio of the average power of the desired signal $\left(P_{d}\right)$ divided by the average power of the undesired signal $\left(P_{i+n}\right)$. The SINR is selected as the second performance criteria for the assessments and computed as:

$$
\operatorname{SINR}=\frac{P_{d}}{P_{i+n}}=\frac{\sum_{d=1}^{D} \sigma_{d}^{2}\left|w^{H} a_{d}\left(\theta_{d}\right)\right|}{\sum_{i=1}^{I} \sigma_{i}^{2}\left|w^{H} a_{i}\left(\theta_{i}\right)\right|+\sigma_{n}^{2}}
$$

The terms $\sigma_{d}, \sigma_{i}$ and $\sigma_{n}$ denote the real user power, unwanted user power, and noise power.

\section{Results And Discussion}

In this section, the efficiency of the MVDR technique based on parallel antenna array geometry is presented. The four scenarios provided are mostly described in terms of extreme conditions, i.e. by increasing the number of array elements $(L)$ over steering the main beam toward the specified direction $\theta_{d}$ with rejecting interference presented on the array at $\theta_{i}$. Figure 2 shows four parallel uniform linear array geometries implemented in this paper. 

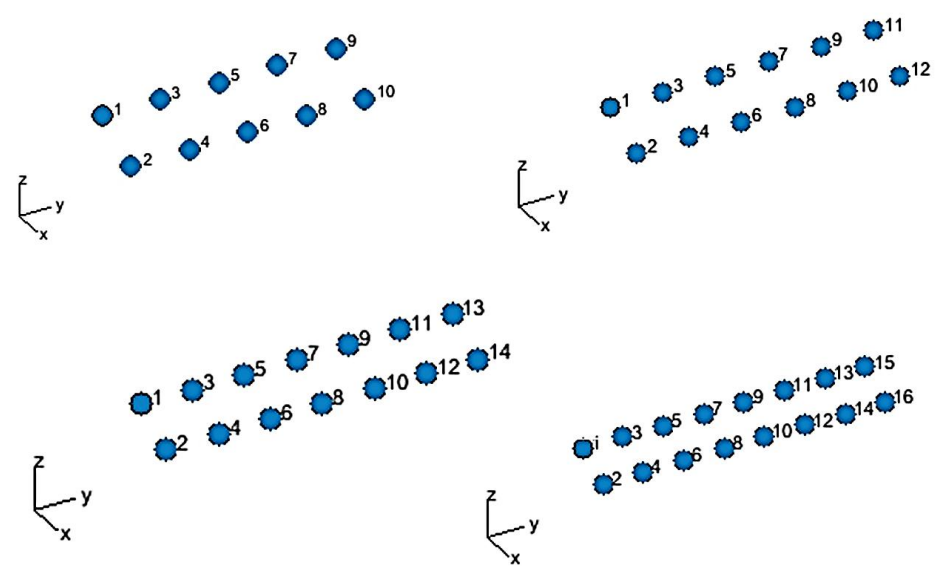

Figure 2: Four scenarios for parallel 2D uniform linear array arrangement.

Table 1 shows the key simulation parameters applied in each scenario. Assume that the inter element spacing $d=d_{N}=d_{M}=\lambda / 2$, where $M$ and $N$ are the inter element space distance in the x-y plane.

Table 1: Main simulation parameters for the MVDR beam former

\begin{tabular}{|l|c|l|}
\hline Main system parameters & Parameter & Values \\
\hline The geometry of the antenna array & --- & Parallel Linear antenna array \\
\hline Kind of Antenna & --- & Isotropic \\
\hline Carrier frequency (Fc) & $F c$ & $2.6 \mathrm{GHz}$ \\
\hline Beam scanning range & $\theta$ & $\pm 90^{\circ}$ (Azimuth) \\
\hline Number of element (L) & $L$ & $10,12,14$ and 16 \\
\hline Element spacing (D) & & $\lambda / 2$ \\
\hline Snapshots (K) & $K$ & 300 \\
\hline Noise power label ( $\sigma \mathrm{n})[\mathrm{dB}]$ & $\sigma_{n}$ & 10 \\
\hline SOI direction & $\theta_{d}$ & $-5^{\circ}$ \\
\hline SNOI direction & $\theta_{i}$ & $-35^{\circ}, 30^{\circ}$ and $60^{\circ}$ \\
\hline SINR target & --- & Max \\
\hline
\end{tabular}

This setting in table 1 aims to check if the given algorithm can steer the main beam towards the desired user while placing the nulls towards interference sources. It has been preferred that at the first the desired user its angle of arrival $\theta_{d}=-5^{\circ}$ while the three interferers signal coming from $\theta i=-35^{\circ}$, $30^{\circ}$, and $60^{\circ}$. The simulation results for $L=10,12,14$, and 16 have been presented in figures 3-7 below. 


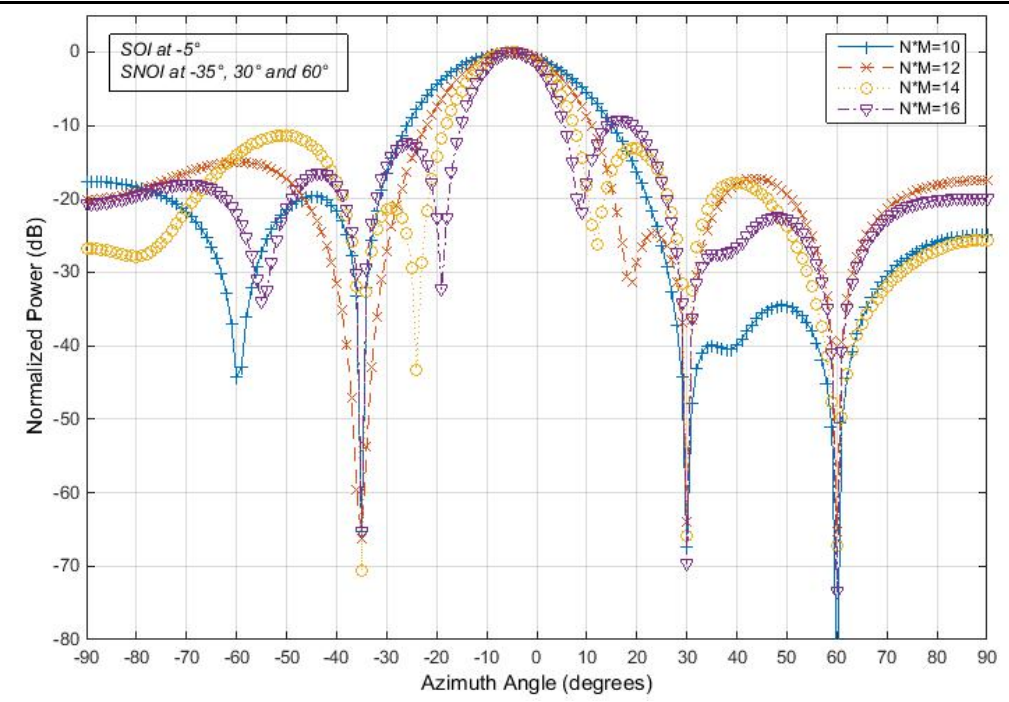

Figure 3: Output beam pattern for the MVDR beam former at $L=10,12,14$, and 16 elements.

The figures demonstrate that the radiation characteristics of the MVDR beamformer concerning the fixed antenna array geometry dimension for a different number of elements. To assess the MVDR beamformer performance applied to the adaptive beamforming problem of the parallel-uniform linear array. The simulation results show transmitting pattern (normalized power response in $\mathrm{dB}$ ) versus the azimuth plane (degrees) throughout figure 3 . It can be seen that the MVDR suppress the interference signals coming from $-35^{\circ}, 30^{\circ}$ and $60^{\circ}$ with a unity gain send to the real user at $-5^{\circ}$. Correspondingly, the polar plot for the MVDR is plotted in figure 4 . The polar plot shows that the mainbeam is directed to the desired user angle and at the same time forming nulls in direction of unwanted user signals. Clearly, it can be realized that the beamwidth of the mainbeam has become narrower as the $L$ increases from 10 to 16 elements. This narrower beam gives less energy consumption by directing the main power to the desired user and no signal transmitted in an unwanted area with less power transmitted in the unwanted direction.

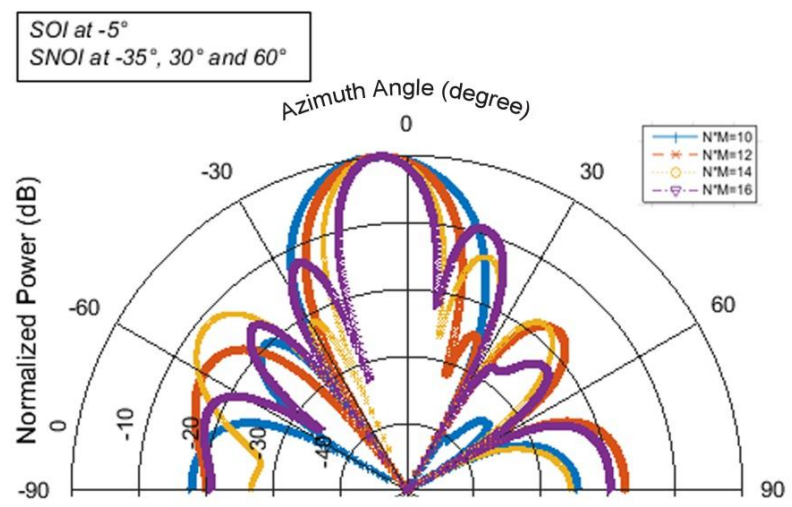

Figure 4: Output beam pattern in polar plot for the MVDR beam former at $L=10,12,14$, and 16 elements. 
International Journal of Intelligent Communication, Computing and Networks

Open Access Journal (ISSN: 2582-7707)

https://doi.org/10.51735/ijiccn/001/36

The resulting weights for each beam former based on a different number of elements are listed in

Table 2. To distinguish between a different number of array elements on the beam former output.

Figure 5 and 6 shows the 3D plot for the beam pattern.

Table 2: Performance analysis of the MVDR beam former output power at $L=10,12,14$, and 16 elements.

\begin{tabular}{|c|c|c|c|c|c|}
\hline \multirow{2}{*}{$L$} & \multicolumn{4}{|c|}{ Power $[\mathrm{dB}]$} & $\operatorname{SINR}[\mathrm{dB}]$ \\
\hline & -5 & -35 & 30 & 60 & Max \\
\hline 10 & 0.05 & -62 & -68 & -83 & 41.3 \\
\hline 12 & 0.03 & -63 & -64 & -65 & 42.9 \\
\hline 14 & 0.02 & -71 & -66 & -67 & 45.1 \\
\hline 16 & 0.01 & -62 & -70 & -74 & 46.6 \\
\hline
\end{tabular}
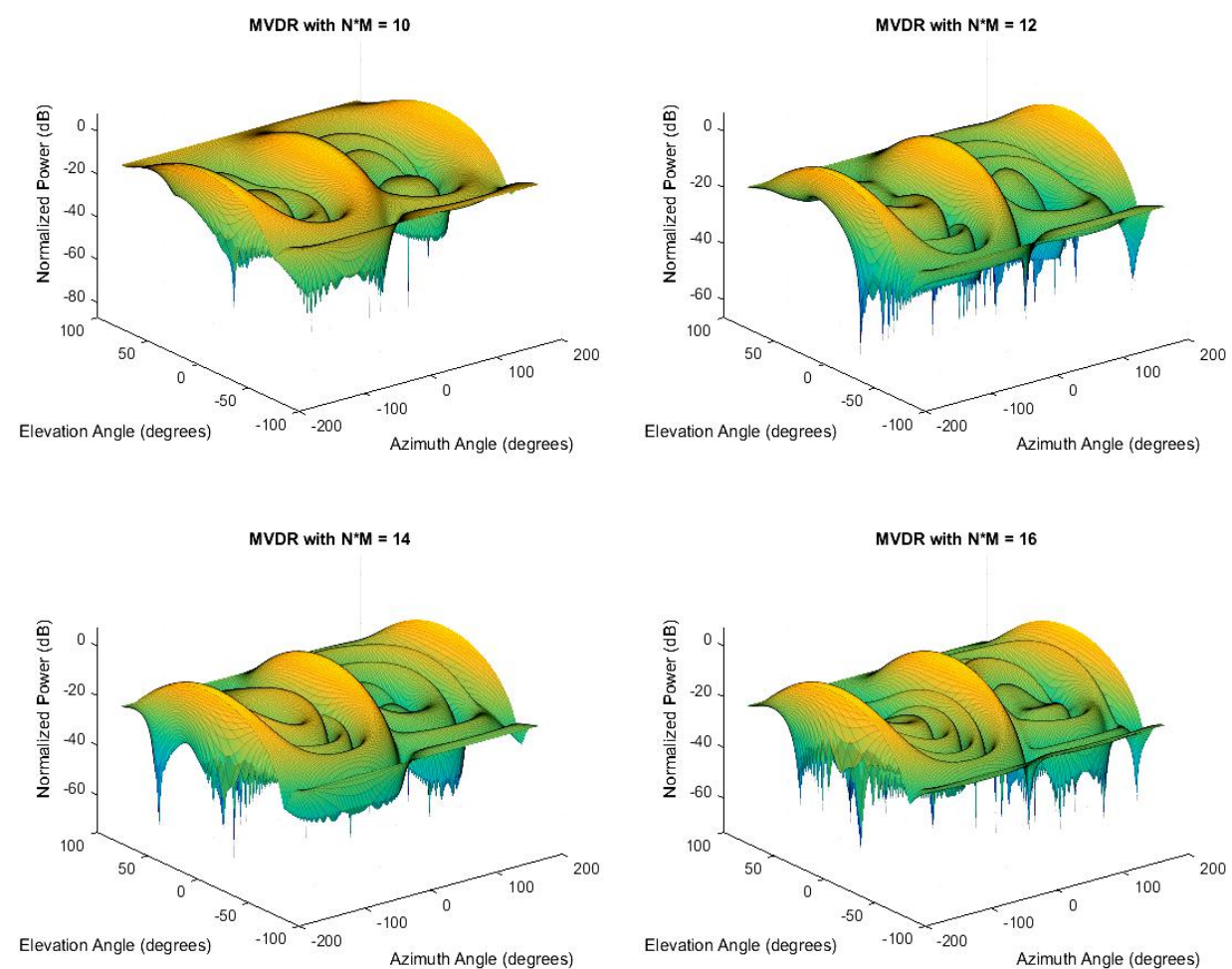

Figure 5: 3D-MVDR beam pattern plot for $L=10,12,14$, and 16 elements. 

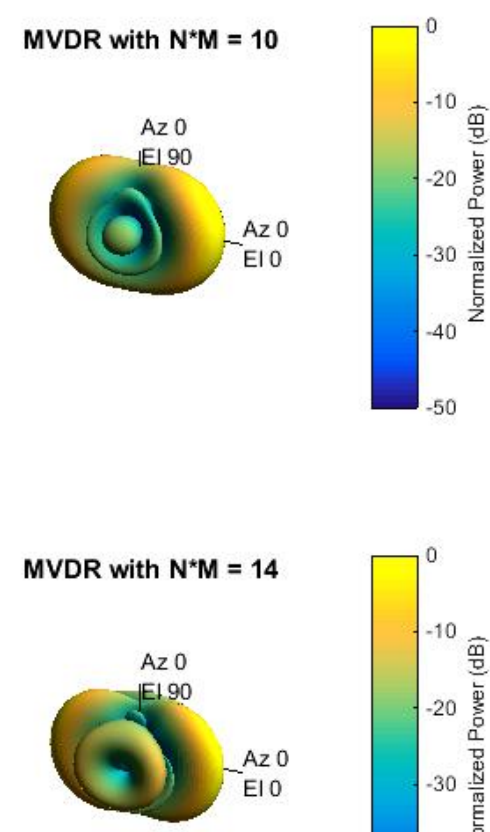
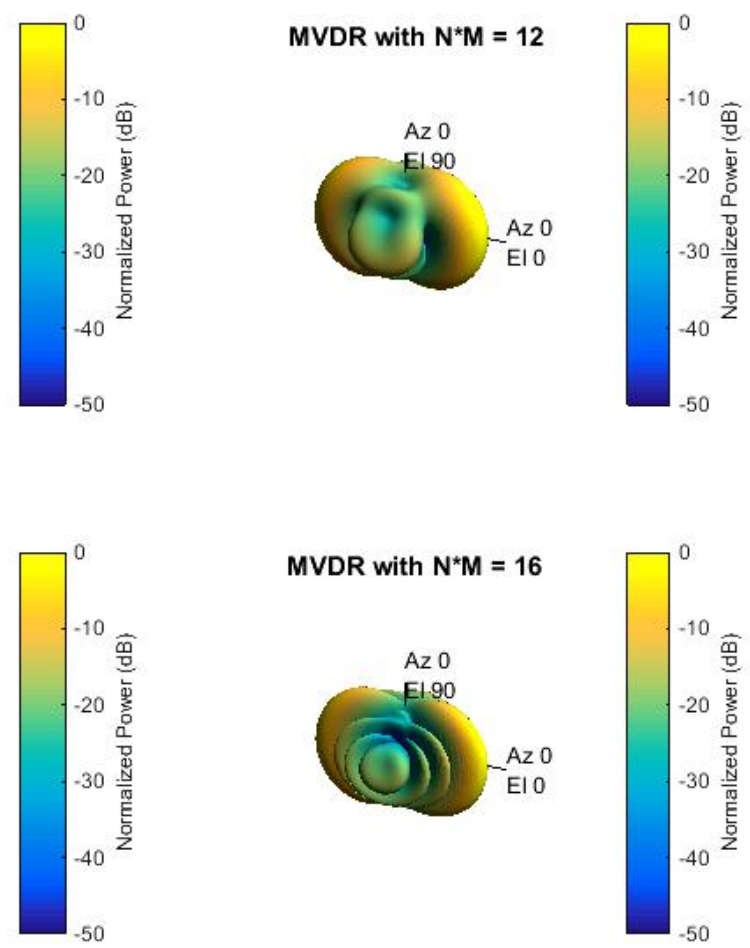

Figure 6: 3D-MVDR beam pattern polar plot for $L=10,12,14$, and 16 elements.

It can be noted that the main beam of the MVDR beam former based on 16 parallel elements is lower than that of others at array geometry $<16$. On contrary, the side lobe level of the MVDR beam former based on a parallel uniform linear array achieves the lowest side lobe level at $\lambda / 2$. In general, the main beam is always directed for look angle based on the MVDR beam former technique and placing nulls to reach the unwanted signals. From figure 7 , it is clear that from the 3D plot the narrower beam was achieved with 16 elements and this is due to the limited number of the antenna array, and antenna array geometry. In beam formers based MVDR algorithm, as array $L$ increases, the mean beam steered to the desired direction of SOI. This conforms to the assumption of MVDR beam former of constrain by sending unity gain in the desired user angle, and the array weights values forming null in the undesired sources. It can be shown how effectively the MVDR technique based on parallel linear array geometry achieves the lowest interference rejection level, and its SINR between $41 \mathrm{~dB}$ to $46 \mathrm{~dB}$ at $10<$ $L<16$. Clearly, transmitting radiation pattern-based MVDR beam former is not deviated from the desired direction $\theta_{s}=-5^{\circ}$. 

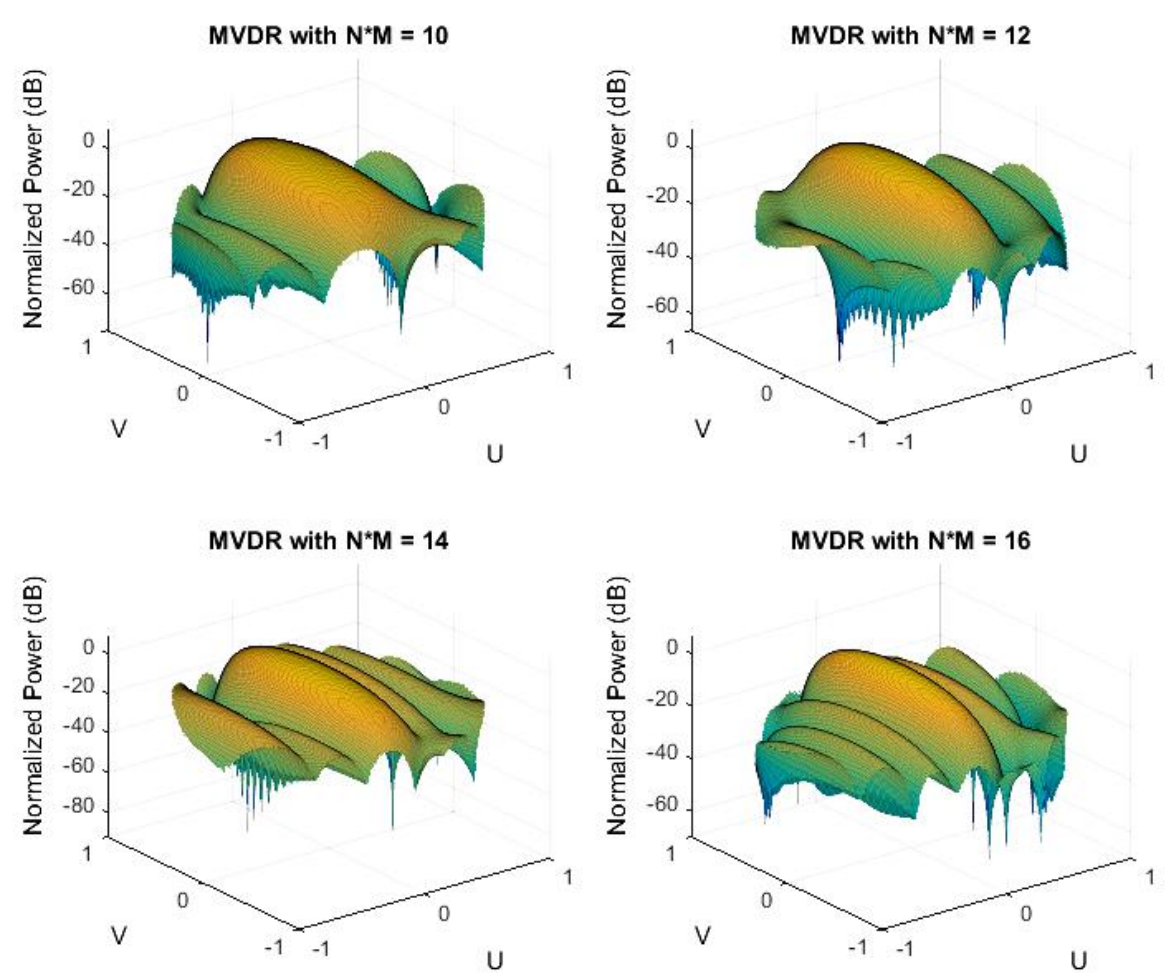

Figure 7: 3D-MVDR beam pattern plot (zoom in) for $L=10,12,14$, and 16 elements.

\section{Conclusion}

Nowadays, one of the most important concerns of wireless communication systems is building accurate beam patterns in smart antennas. Beam forming technology forms beam toward look angle at the same time placing null toward inferences signals. This paper has underlined the importance of using beam forming technology in wireless telecommunication. The present work studied the MVDR beam former behavior based on two uniform linear arrays placed in parallel. The simulation results confirmed that the MVDR technique based on parallel uniform linear antenna array geometry can direct the main lobe to the desired user angle while forming null to the undesired sources. The present findings have important implications for mitigating multiple interference sources. Results so far indicate that as the number of elements increases in the antenna array, narrower main lobe beam width and $L-1$ null can be achieved. It is found $1 \mathrm{~dB}$ increases in the SINR value as one element is added to the parallel uniform linear array. It can be concluded that the MVDR beam former on antenna array using a two linear array is certainly possible.

\section{Acknowledgments}

This work is supported by Al Hawija Technical Institute, Northern Technical University, Kirkuk, Iraq.

\section{References}

1. Cisco. (2020). Cisco annual internet report (2018-2023): white paper. Available: https://www.cisco.com/c/en/us/solutions/collateral/executive-perspectives/annual-internet-report/whitepaper-c11 
$-741490 . \mathrm{html}$

2. Thakur, R. K. (2021). Internet of Things (IoT) Security: Present Status and Future Direction. International Journal of Intelligent Communication, Computing and Networks, vol. 2, no. 2, pp. 36-42.

3. Satrusallya, S. (2021). Evaluation of Beam Forming Capability of Linear Antenna Array for Smart Antenna System. Turkish Journal of Computer and Mathematics Education (TURCOMAT), vol. 12, no. 9, pp. 637-642.

4. Nilofera, S. (2020). A Review of Massive Multiple Input Multiple Output for 5G Communication: Benefits and Challenges. International Journal of Intelligent Communication, Computing and Networks, vol. 1, no. 1, pp. 22-26, 2020.

5. Filik T. and Tuncer, T. (2011). 2-D paired direction-of-arrival angle estimation with two parallel uniform linear arrays. International Journal of Innovative Computing, Information and Control, vol. 7, no. 6, pp. 3269-3279.

6. Zhang D., Zhang, Y. and Feng, C. (2018). Enhanced 2D-DOA Estimation for Large Spacing Three-Parallel Uniform Linear Arrays. International Journal of Antennas and Propagation, vol. 2018.

7. Chen, L., Bi, D., \& Pan, J. (2018). Two-dimensional angle estimation of two-parallel nested arrays based on sparse Bayesian estimation. Sensors, vol. 18, no. 10, p. 3553.

8. Shahab, S. N., Zainun, A. R., Noordin, N. H., \& Balasim, S. S. (2016). Assessment of MVDR Adaptive Beamforming Algorithm in Uniform Linear Arrays, Uniform Rectangular Arrays and Uniform Circular Arrays Configurations. APRN Journal of Engineering and Applied Sciences, vol. 11, pp. 3911-1917.

9. Shahab, S. N., Zainun, A. R., Ali, H. A., Hojabri, M., \& Noordin, N. H. (2017). MVDR algorithm based linear antenna array performance assessment for adaptive beamforming application. Journal of Engineering Science and Technology, vol. 12, no. 5, pp. 1366-1385.

10. Shahab, S. N., Zainun, A. R., Noordin, N. H., Mohamad, A. J., \& Khaldoon, O. (2015). Performance analysis of smart antenna based on MVDR beamformer using rectangular antenna array. ARPN Journal of Engineering and Applied Sciences, , vol. 10, no. 22, pp. 17132-17138.

11. Shahab, S. N., Zainun, A. R., Balasim, S. S., Noordin, N. H., \& Ibrahim, I. (2017). Evaluation of Minimum Variance Distortionless Response Beamforming Algorithm Based Circular Antenna Arrays. Modern Applied Science, vol. 11, no. 1, pp. 1-13.

12. Nagaraju, V., Sethy, A., Boddu, R. S. K., Balambigai, S., \& Sakthisudhan, K. (2021). Diagnosed image of breast cancer by antenna MVDR beamforming algorithm with composites. Materials Today: Proceedings, pp. In Press.

13. Zhang, Z., Xu, Y., Yu, M., Zhang, S. X., Chen, L., \& Yu, D. (2021). ADL-MVDR: All deep learning MVDR beamformer for target speech separation. In ICASSP 2021 IEEE International Conference on Acoustics, Speech and Signal Processing (ICASSP), pp. 6089-6093.

14. Ramezanpour, P., Rezaei, M. J., \& Mosavi, M. R. (2020). Deep-learning-based beamforming for rejecting interferences. IET Signal Processing, vol. 14, no. 7, pp. 467-473.

15. Godara, L. C. (2004). Smart antennas. Boca Raton: CRC press. 